\title{
Relationships between Cognitive Function and Odor Identification, Balance Capability, and Muscle Strength in Middle-Aged Persons with and without Type 2 Diabetes
}

\author{
Manabu Midorikawa, ${ }^{1,2}$ Hiroaki Suzuki (iD), ${ }^{2}$ Yasuhiro Suzuki, ${ }^{3}$ Kazuyoshi Yamauchi, ${ }^{4}$ \\ Hiroyuki Sato, ${ }^{1,5}$ Kiyotaka Nemoto, ${ }^{5}$ Yoko Sugano, ${ }^{2}$ Hitoshi Iwasaki, ${ }^{2}$ Motohiro Sekiya, ${ }^{2}$ \\ Shigeru Yatoh, ${ }^{2}$ Naoya Yahagi, ${ }^{2}$ Yasushi Hada, ${ }^{6}$ Tetsuaki Arai, ${ }^{5}$ and Hitoshi Shimano ${ }^{2,7,8,9}$ \\ ${ }^{1}$ Doctoral Program in Clinical Sciences, Graduate School of Comprehensive Human Sciences, University of Tsukuba, \\ Tsukuba 305-8575, Japan \\ ${ }^{2}$ Department of Internal Medicine (Endocrinology and Metabolism), Faculty of Medicine, University of Tsukuba, \\ Tsukuba 305-8575, Japan \\ ${ }^{3}$ Department of Rehabilitation Medicine, University of Tsukuba Hospital, Tsukuba 305-8596, Japan \\ ${ }^{4}$ Department of Laboratory Medicine, Faculty of Medicine, University of Tsukuba, Tsukuba 305-8575, Japan \\ ${ }^{5}$ Department of Psychiatry, Faculty of Medicine, University of Tsukuba, Tsukuba 305-8575, Japan \\ ${ }^{6}$ Department of Rehabilitation Medicine, Faculty of Medicine, University of Tsukuba, Tsukuba 305-8575, Japan \\ ${ }^{7}$ International Institute for Integrative Sleep Medicine (WPI-IIIS), University of Tsukuba, Tsukuba 305-8575, Japan \\ ${ }^{8}$ Life Science Center of Tsukuba Advanced Research Alliance (TARA), University of Tsukuba, Tsukuba 305-8575, Japan \\ ${ }^{9} J a p a n$ Agency for Medical Research and Development-Core Research for Evolutional Science and Technology (AMED-CREST), \\ Chiyoda-ku 100-0004, Japan
}

Correspondence should be addressed to Hiroaki Suzuki; hirosuzu@md.tsukuba.ac.jp

Received 28 March 2021; Accepted 7 September 2021; Published 7 October 2021

Academic Editor: Cesare Patrone

Copyright () 2021 Manabu Midorikawa et al. This is an open access article distributed under the Creative Commons Attribution License, which permits unrestricted use, distribution, and reproduction in any medium, provided the original work is properly cited.

\begin{abstract}
Aim. We investigated the relationship between cognitive function and olfactory and physical functions in middle-aged persons with and without type 2 diabetes (T2D) to examine the potential of olfactory and physical functions as biomarkers for early cognitive impairment. Methods. Enrolled were 70 T2D patients (age 40 to <65y) and 81 age-matched control participants without diabetes. Cognitive function was assessed by the Montreal Cognitive Assessment (MoCA), Trail Making Test parts A and B (TMT-A/-B), Wisconsin Card Sorting Test (WCST), Quick Inventory of Depressive Symptomatology Self-Report (QIDS), and Starkstein Apathy Scale (SAS). Multiple linear regression analyses were performed. Results. Odor identification was an independent determinant shown in the results of the TMT-A in the entire participant group and was independently associated with the MoCA and TMT-B in the T2D group. Balance capability assessed with a stabilometer was independently associated with all cognitive function tests except for QISD and SAS in the entire participant group and the T2D group and was independently associated with TMT-A in the control group. Knee extension strength was independently associated with the SAS in the entire participant group and the T2D group. Conclusions. Odor identification, balance capability, and knee extension strength were potential markers for cognitive decline in middle-aged persons with T2D.
\end{abstract}




\section{Introduction}

The increase in dementia and its prevention and treatment are global concerns [1]. Although several drugs have been developed to improve cognitive decline in Alzheimer's disease $(\mathrm{AD})$ [2], none has proven to be sufficiently effective [2]. It was reported that $16.5 \%$ of people with mild cognitive impairment (MCI), which is a precursor to dementia, develop $\mathrm{AD}$ annually [3], while about $20 \%$ recover to normal cognitive function [4]. Therefore, preventive interventions for persons with MCI or normal cognitive function who are at risk of developing dementia are considered to be important for dementia prevention.

Several modifiable risk factors for dementia have been reported, with diabetes being one of them [5]. In a systematic review of investigations of the development of dementia in persons with diabetes, the relative risk was 1.7-2.2 for $\mathrm{AD}$ and 2.2-2.8 for vascular dementia $(\mathrm{VaD})$ [6]. Acute fluctuations of plasma glucose levels present a risk for dementia: elevated levels of 2 -hour postload glucose in a $75 \mathrm{~g}$ oral glucose tolerance test were associated with the development of all-cause dementia, $\mathrm{AD}$, and $\mathrm{VaD}$ in a population-based study [7], daily acute glucose fluctuations assessed with continuous glucose monitoring were reported to be independently associated with cognitive impairment in persons with type 2 diabetes (T2D) [8], and a cross-sectional study showed that visit-to-visit glucose variability was independently associated with the Mini-Mental State Examination score in elderly persons with T2D [9]. Although increased $\mathrm{HbAlc}$ levels and hypoglycemia have been thought to be risk factors for cognitive decline in diabetes, results of a systematic review were inconsistent [10]. In addition, cardiometabolic risk factors such as hypertension, dyslipidemia, and obesity, which are often associated with diabetes, are risk factors for cognitive decline in the general population [11].

Numerous studies have reported on associations between olfactory or physical dysfunction and cognitive decline. Olfactory dysfunction, especially odor identification, was observed in persons with $\mathrm{MCI}$ and $\mathrm{AD}[12,13]$ and was more prominent in AD compared with $\mathrm{MCI}[13,14]$. The results of an odor identification score were significantly associated with $\mathrm{AD}$ pathology based on counts of cortical plaques and tangles or density of tau-positive neurofibrillary tangles in the entorhinal cortex and hippocampus [15]. With regard to physical function, both gait speed and balance capability were significantly associated with cognitive function in elderly people [16]. A systematic review showed that changes in grip strength were associated with changes in cognitive function, although this association was inconsistent [17]. Impaired lower extremity function assessed by walking speed and the number of steps taken within a designated time, standing up from a chair, and balancing was significantly associated with the development of $\mathrm{AD}$ in persons with $\mathrm{MCI}$, whereas there was no significant association between dexterity and the development of AD [18]. Furthermore, olfactory function was shown to be associated with motor function in elderly persons [19].

Relationships among cognitive function, olfactory function, and physical function have also been reported in those with diabetes. A systematic review showed that persons with diabetes had significant olfactory impairment compared with control participants [20]. Furthermore, the olfactory identification score was an independent determinant of cognitive impairment assessed with the Mini-Mental State Examination in elderly persons with T2D [21]. Both timed walk and grip strength were independent determinants of performance in all cognitive domains except for episodic memory in elderly persons with T2D [22].

A prospective cohort study showed that a younger onset of T2D was significantly associated with an increased risk of dementia: at the age of 70 years, the hazard ratio of dementia for every 5-year increment of earlier onset of T2D was 1.24 [23]. Therefore, interventions that take cognitive decline into account are important in middle-aged persons with T2D and early detection of persons with T2D who are at higher risk of cognitive decline may be useful in devising strategies to prevent or delay the subsequent onset of dementia. Although the identification of noninvasive biomarkers, such as olfactory function and physical function, is important for the early prevention of cognitive decline in persons with T2D, the relationship between cognition and olfactory function and physical function in middle-aged persons with T2D has not been reported. Nor has such relationships been reported in middle-aged persons without diabetes. The clinical questions in the current study were (i) whether olfactory function, balance capability, and muscle strength were associated with cognitive function even in the middle-aged adults, (ii) whether those associations were different between persons with diabetes and those without diabetes, and (iii) which physical function was important for each cognitive domain.

It must be recognized that testing methods used in studies of the elderly of balance capability using one-leg standing time (OLST) or the Timed Up and Go Test (TUG) and muscle strength testing using hand-held dynamometry may be so easy for middle-aged persons that subtle differences among groups or participants cannot be detected through these means. We aimed at investigating the relationships between cognitive function and olfactory function and physical function in middle-aged persons with T2D and nondiabetic controls to clarify the potential of these factors as biomarkers for early detection of cognitive impairment. In addition, we examined whether precise balance and muscle strength assessments using a stabilometer and a torque machine could be useful in predicting cognitive dysfunction in middle-aged adults with and without T2D.

\section{Methods}

2.1. Participants. From November 2017 to August 2020, we enrolled 70 persons with T2D and 81 nondiabetic control participants who were 40 to $<65$ years old and had no clinically apparent cognitive impairment. The diabetic persons were recruited from those who had visited outpatient clinics or were admitted to hospital for treatment of diabetes. Nondiabetic control participants were recruited using our department's website, bulletin boards at our university, and a local community magazine. Exclusion criteria included 
(i) history of central nervous system disorders, head injury, psychiatric disorders, or olfactory disorders; (ii) rhinitis; (iii) use of drugs affecting neuropsychological function; and (iv) being unable to walk independently without assistive devices. No one was excluded based on results of cognitive function tests. Written informed consent was obtained from participants prior to study enrollment. This study was conducted in accordance with the principles of the Declaration of Helsinki and approved by the Clinical Research Ethics Committee of University of Tsukuba Hospital (H29129).

2.2. Clinical Evaluation. Participants were surveyed for age, sex, smoking habits, years of education, systolic blood pressure, diastolic blood pressure, physical activity, and medication status. In addition, in persons with T2D, information was obtained on the duration of diabetes and diabetic complications. All participants were asked about the frequency, amount, and type of alcohol that they currently consumed. The amount of physical activity was calculated using the International Standardized Physical Activity Questionnaire [24]. Body composition was evaluated by bioelectrical impedance analysis (InBody 720, BioSpace, Tokyo). Body mass index (BMI) and skeletal muscle mass index were calculated by dividing the body weight $(\mathrm{kg})$ by the square of the height $\left(\mathrm{m}^{2}\right)$ and dividing the limb skeletal muscle mass $(\mathrm{kg})$ by the square of the height $\left(\mathrm{m}^{2}\right)$, respectively.

2.3. Assessment of Cognitive Function. Global cognitive function was assessed using the Japanese version of the Montreal Cognitive Assessment (MoCA) [25]. Originally, participants with $<12$ years of education would receive an additional 1 point on the MoCA score to assess cognitive function; however, we used raw data because age-adjusted scores were reported to be less sensitive in detecting cognitive decline [26]. Trail Making Test parts A and B (TMT-A/-B) were used for assessing processing speed and executive function, respectively [27]. The personal computer version of the Wisconsin Card Sorting Test Keio version (KWCST) [28], which is a simplified version of the Wisconsin Card Sorting Test (WCST), was used to assess frontal lobe functions such as cognitive flexibility and abstract concepts. The number of categories achieved (CA) and perseverative errors of the Nelson type (PEN) were extracted. Depressive symptoms were assessed using the Japanese version of the Quick Inventory of Depressive Symptomatology Self-Report (QIDS) [29]. The Japanese version of the Starkstein Apathy Scale (SAS) was used to evaluate motivation [30].

2.4. Assessment of Olfactory Function. Olfactory function was evaluated using a card-type odor identification test developed for Japanese people (Open Essence ${ }^{\circledR}$, Fuji Film Wako Pure Chemicals, Tokyo, Japan). Open Essence consists of 12 kinds of odorants, which are perfume, rose, condensed milk, mandarin orange, curry, roasted garlic, sweatsmelling clothes, cooking gas, menthol, India ink, wood, and Japanese cypress [31]. Total scores ranged from 0 to 12 points.
2.5. Assessment of Physical Function. Balance capability was evaluated with the OLST, TUG, and index of postural stability (IPS). OLST was measured with both eyes open and also with both eyes closed. The test was performed twice with the examinee standing on each leg, and the longest time was considered to be the representative value. The maximum measurement time was 120 seconds with the eyes open and 60 seconds with the eyes closed. TUG was performed as follows: the participant stood up from a chair without elbow rests and walked 3 meters forward and back as quickly as possible [32]. IPS was measured using a stabilometer (GP6000, Anima, Tokyo, Japan) as described elsewhere [33]. First, participants stood in a resting position with the inside of the foot at a distance of $10 \mathrm{~cm}$ on the stabilometer to measure instantaneous fluctuations in the center of pressure (COP) at a sampling frequency of $20 \mathrm{~Hz}$. Then, participants were instructed to incline the body to the front, rear, right, and left keeping the body straight and without moving the feet. The instantaneous fluctuations in COP were measured at each position. IPS was calculated as "log [(area of stability limit + area of postural sway)/area of postural sway]." The area of the stability limit was calculated as the "front and rear center movement distance between anterior and posterior positions $\times$ the distance between right and left positions." The area of postural sway was calculated as "average measurement value in 10 seconds under anterior, posterior, right, left, and center positions." The area of postural sway was calculated as the mean sway area of the 5 positions.

Muscle strength was evaluated according to grip strength, knee extension muscle strength, and knee extension muscle endurance. The grip strength of the dominant hand was measured using a Smedley analog grip strength meter (Toei Light, Saitama, Japan). Knee extension muscle strength and knee extension muscle endurance were evaluated on the dominant foot side using a torque machine (Biodex System 3, Sakai Medical, Tokyo, Japan). For knee extension muscle strength, the participant performed three consecutive knee extension operations with maximum effort in isokinetic muscle strength measurement $\left(60^{\circ} / \mathrm{s}\right)$ and the maximum torque value adjusted by body weight was used as a representative measurement value. Knee extension muscle endurance was measured by the total work from 20 continuous knee extensions with maximum effort by isokinetic muscle strength measurements $\left(300^{\circ} / \mathrm{s}\right)$.

2.6. Laboratory Analysis. Blood samples were collected in the morning after an overnight fast. Plasma glucose and total cholesterol, low-density lipoprotein cholesterol, high-density lipoprotein cholesterol, and triglycerides were determined using an automated analyzer (Hitachi High-Technologies, Tokyo, Japan). HbAlc was measured by high-performance liquid chromatography (TOSOH, Tokyo, Japan). The apolipoprotein $\mathrm{E}$ (ApoE) genotype was analyzed by a polymerase chain reaction-restriction fragment length polymorphism method [34].

2.7. Statistical Analysis. Based on distribution, continuous variables were expressed as mean \pm standard deviation or 
TABLE 1: Clinical characteristics of study participants.

\begin{tabular}{|c|c|c|c|c|}
\hline & All $(\mathrm{n}=151)$ & Controls $(\mathrm{n}=81)$ & Type 2 diabetes $(\mathrm{n}=70)$ & $P$ \\
\hline Age (years) & $53 \pm 7$ & $52 \pm 6$ & $53 \pm 7$ & 0.323 \\
\hline Female, n (\%) & $75(50)$ & $47(58)$ & $28(40)$ & 0.020 \\
\hline Education (y) & $14(12-16)$ & $15(14-16)$ & $12(12-14)$ & $<0.001$ \\
\hline ApoE $\varepsilon 4$ allele carrier, $\mathrm{n}(\%)$ & $29(19)$ & $22(27)$ & $7(10)$ & 0.006 \\
\hline Current Smoking, n (\%) & $25(17)$ & $5(6)$ & $20(29)$ & $<0.001$ \\
\hline Alcohol consumption (g/d) & $6(0-20)$ & $7(0-20)$ & $0(0-20)$ & 0.092 \\
\hline Total physical activity (MET-min/w) & $1724(656-4064)$ & $2010(735-4299)$ & $1253(401-3803)$ & 0.098 \\
\hline Body mass index $\left(\mathrm{kg} / \mathrm{m}^{2}\right)$ & $24.6(21.9-28.4)$ & $22.1(21.1-24.5)$ & $28.2(25.4-30.1)$ & $<0.001$ \\
\hline Body fat percentage (\%) & $29.9 \pm 8.8$ & $26.6 \pm 7.7$ & $33.8 \pm 8.4$ & $<0.001$ \\
\hline Skeletal muscle percentage (\%) & $38.4 \pm 5.3$ & $40.3 \pm 4.7$ & $36.3 \pm 5.1$ & $<0.001$ \\
\hline Skeletal muscle mass index $\left(\mathrm{kg} / \mathrm{m}^{2}\right)$ & $7.2(6.4-8.0)$ & $6.9(6.1-7.6)$ & $7.6(6.7-8.5)$ & $<0.001$ \\
\hline Fasting plasma glucose (mmol/L) & $5.8(5.1-8.7)$ & $5.1(4.9-5.4)$ & $8.9(7.7-10.3)$ & $<0.001$ \\
\hline HbAlc (\%) & $6.0(5.6-8.5)$ & $5.6(5.4-5.8)$ & $8.9(7.6-11.1)$ & $<0.001$ \\
\hline $\mathrm{HbA1c}(\mathrm{mmol} / \mathrm{mol})$ & $42(38-69)$ & $38(36-40)$ & $74(60-98)$ & $<0.001$ \\
\hline Total cholesterol (mmol/L) & $5.2 \pm 0.9$ & $5.5 \pm 0.7$ & $4.9 \pm 1.0$ & $<0.001$ \\
\hline HDL-C (mmol/L) & $1.6 \pm 0.5$ & $1.9 \pm 0.4$ & $1.2 \pm 0.2$ & $<0.001$ \\
\hline LDL-C (mmol/L) & $3.0 \pm 0.7$ & $3.1 \pm 0.6$ & $3.0 \pm 0.8$ & 0.618 \\
\hline Triglycerides (mmol/L) & $1.1(0.8-1.7)$ & $0.8(0.7-1.1)$ & $1.7(1.2-2.3)$ & $<0.001$ \\
\hline Systolic blood pressure (mmHg) & $127(116-138)$ & $125(115-135)$ & $129(118-142)$ & 0.084 \\
\hline Diastolic blood pressure $(\mathrm{mmHg})$ & $80 \pm 12$ & $81 \pm 12$ & $79 \pm 12$ & 0.450 \\
\hline Hypertension, n (\%) & $35(23)$ & $7(9)$ & $28(40)$ & $<0.001$ \\
\hline Dyslipidemia, n (\%) & $48(32)$ & $3(4)$ & $45(64)$ & $<0.001$ \\
\hline Duration of diabetes $(y)$ & & & $7.0(2.0-11.0)$ & \\
\hline \multicolumn{5}{|l|}{ Diabetic complications } \\
\hline Retinopathy, n (\%) & & & $18(26)$ & \\
\hline Nephropathy, n (\%) & & & $23(33)$ & \\
\hline Peripheral neuropathy, n (\%) & & & $26(37)$ & \\
\hline Cardiovascular disease, $\mathrm{n}(\%)$ & & & $8(11)$ & \\
\hline \multicolumn{5}{|l|}{ Antidiabetic drugs } \\
\hline Metformin, n (\%) & & & $43(61)$ & \\
\hline Sulfonylureas, n (\%) & & & $15(21)$ & \\
\hline Glinides, n (\%) & & & $1(1)$ & \\
\hline Thiazolidinediones, n (\%) & & & $7(10)$ & \\
\hline Sodium-glucose cotransporter 2 inhibitors, $\mathrm{n}(\%)$ & & & $22(31)$ & \\
\hline Dipeptidyl peptidase- 4 inhibitors, $\mathrm{n}(\%)$ & & & $33(47)$ & \\
\hline Glucagon like peptide-1receptor agonists, n (\%) & & & $6(9)$ & \\
\hline Alpha-glucosidase inhibitors, n (\%) & & & $3(4)$ & \\
\hline Insulin, $\mathrm{n}(\%)$ & & & $16(23)$ & \\
\hline
\end{tabular}

Data are mean \pm SD or median (interquartile range). ApoE: apolipoprotein E; HDL-C: high-density lipoprotein-cholesterol; LDL-C: low-density lipoproteincholesterol.

median (interquartile range) and compared using the unpaired $t$-test or the Mann-Whitney $U$-test for twogroup comparisons. Categorical variables are expressed as numerals and percentages and were compared with Fisher's exact test. Spearman's rank correlation coefficient was used to examine bivariate associations between tests of cognitive function and olfactory or physical functions. A multiple linear regression analysis was performed to examine whether olfactory function, or physical functions were independent determinants of cognitive functions. We adopted IPS and knee extension strength as explanatory variables representative of balance capability and muscle strength based on results of the correlation analysis in the overall, T2D, and control groups. TMT-A, TMT-B, PEN in KWCST, and QIDS were log transformed, and MoCA was cubed due to their nonnormal distribution. Because the scores of PEN and QIDS contain 0, we added 1 to the scores of PEN and QIDS before log transformation. The ApoE genotype was categorized as either an $\varepsilon 4$ carrier or noncarrier. Statistical analyses were performed using SPSS Statistics 26 (Chicago, 
TABLE 2: Comparison of cognitive function tests, odor identification test, and physical function tests.

\begin{tabular}{lccc}
\hline & All $(\mathrm{n}=151)$ & Controls $(\mathrm{n}=81)$ & Type 2 diabetes $(\mathrm{n}=70)$ \\
\hline Cognitive function & & & \\
MoCA (points) & $26(24-28)$ & $27(26-28)$ & $25(22-27)$ \\
TMT-A (s) & $29.4(23.0-34.6)$ & $25.9(21.4-31.9)$ & $31.7(25.8-39.0)$ \\
TMT-B (s) & $66.1(55.1-90.4)$ & $63.3(52.6-80.7)$ & $79.3(60.0-109.0)$ \\
WCST & & & 0.001 \\
CA (points) & $4(2-5)$ & $4(3-5)$ & $3(1-5)$ \\
PEN (points) & $5(2-12)$ & $3(2-7)$ & $9(4-15)$ \\
QIDS (points) & $4(2-7)$ & $3(2-6)$ & $5(3-9)$ \\
SAS (points) & $11 \pm 6$ & $9 \pm 5$ & $13 \pm 6$ \\
Olfactory function & $9(8-10)$ & $10(8-11)$ & $<0.001$ \\
Open Essence (points) & & & $<(8-10)$ \\
Physical function & $120(86-120)$ & $120(120-120)$ & $<0.001$ \\
OLST (eyes open) (s) & $13(6-28)$ & $21(10-39)$ & $<0.001$ \\
OLST (eyes closed) (s) & $5.5 \pm 1.0$ & $5.0 \pm 0.7$ & $<(37-120)$ \\
TUG (s) & $1.80(1.63-1.96)$ & $1.92(1.81-2.02)$ & $7(4-14)$ \\
IPS & $31(25-41)$ & $31(27-41)$ & $6.1 \pm 0.8$ \\
Grip strength (kg) & $190 \pm 49$ & $211 \pm 44$ & $1.64(1.47-1.76)$ \\
Knee extension strength (Nm/kg) & $1053(806-1353)$ & $1081(896-1415)$ & $29(24-41)$ \\
Knee extension endurance (J) & & $166 \pm 43$ \\
\hline
\end{tabular}

Data are mean \pm SD or median (interquartile range). CA: categories achieved; IPS: index of postural stability; MoCA: Montreal Cognitive Assessment; OLST: one-leg standing time; PEN: perseverative errors of Nelson; QIDS: Quick Inventory of Depressive Symptomatology Self-Report; SAS: Starkstein Apathy Scale; TMT-A: Trail Making Test part A; TMT-B: Trail Making Test part B; TUG: Timed Up and Go Test; WCST: Wisconsin Card Sorting Test.

IL, USA). Statistical significance was considered at a $P$ value of $<0.05$.

\section{Results}

Table 1 shows the clinical characteristics of the study participants. The T2D group had significantly higher proportions of smokers, hypertension, and dyslipidemia; significantly lower proportions of females and ApoE $\varepsilon 4$ allele carriers; and significantly shorter education periods compared with the control group. Age, total physical activity, and alcohol consumption were not significantly different between the two groups. As to body composition, BMI, body fat percentage, and SMI were significantly higher and skeletal muscle percentage was significantly lower in the T2D group than in the control group. Table 2 shows the results of cognitive function tests, odor identification test, and physical function tests. Results of cognitive function testing and tests of knee extension strength, endurance, and balance capability in the T2D group were significantly worse than those in the control group. Grip strength did not differ significantly between the two groups.

The results of the correlation analysis between the cognitive function tests and odor identification test, balance capability, and muscle strength were as follows: (i) the odor identification score was significantly correlated with MoCA, TMT-A, and QIDS (Table 3) and with MoCA and TMT-B in the T2D group (Table 4). None of the cognitive subdomains was correlated with the odor identification score in the control group (Table 5), (ii) balance capability-related variables were significantly correlated with all of the cognitive subdomains in the entire cohort (Table 3), with MoCA, TMT-A and -B, CA, and PEN in WCST in the T2D group (Table 4) and with a few cognitive subdomains in the control group (Table 5), and (iii) muscle strength-related variables had significant correlations with all of the cognitive subdomains except for MoCA in the entire cohort (Table 3), with TMT-A and -B, and CA, and PEN in WCST in the T2D group (Table 4) and with TMT-B, PEN in WCST, and SAS in the control group (Table 5).

In the entire participant group, the results of multiple linear regression analyses adjusted by age, sex, education ( $\leq 12$ years or $>12$ years), presence of diabetes, hypertension, ApoE $\varepsilon 4$ carrier, and BMI were as follows (Table 6): (i) results of the odor identification test were significantly associated with TMT-A and tended to be associated with MoCA and QIDS, (ii) IPS was independently associated with all cognitive function tests except for QIDS and SAS, and (iii) knee extension strength was significantly associated with TMT-B and SAS. Table 7 shows the results of multiple linear regression analysis adjusted for age, sex, education, and ApoE $\varepsilon 4$ carrier status in the control group and the T2D group. In the control group, IPS was independently associated with TMT-A and tended to be associated with MoCA and SAS. However, the odor identification test and tests of knee extension strength did not show a statistically significant correlation with any of the cognitive function test results in the control group. In contrast, in the T2D group, results of the odor identification test had independent associations with MoCA and TMT-B, IPS was independently 
TABLE 3: Correlation between cognitive function tests and olfactory identification test, balance capability tests, and muscle strength tests in the entire participants.

\begin{tabular}{|c|c|c|c|c|c|c|c|}
\hline & $\mathrm{MoCA}$ & TMT-A & TMT-B & WCST (CA) & WCST (PEN) & QIDS & SAS \\
\hline Open essence & $0.216^{* *}$ & $-0.191^{*}$ & -0.157 & 0.128 & -0.145 & $-0.210^{*}$ & -0.069 \\
\hline OLST (eyes open) & $0.288^{* * *}$ & $-0.377^{* * *}$ & $-0.286^{* * *}$ & $0.208^{*}$ & $-0.249^{* *}$ & -0.141 & $-0.268^{* * *}$ \\
\hline OLST (eyes closed) & $0.294^{* * *}$ & $-0.326^{* * *}$ & $-0.227^{* *}$ & $0.208^{*}$ & $-0.223^{* *}$ & $-0.252^{* *}$ & $-0.266^{* * *}$ \\
\hline TUG & $-0.275^{* * *}$ & $0.262^{* *}$ & $0.258^{* *}$ & $-0.181^{*}$ & $0.305^{* * *}$ & $0.260^{* *}$ & $0.296^{* * *}$ \\
\hline IPS & $0.442^{* * *}$ & $-0.308^{* * *}$ & $-0.385^{* * *}$ & $0.263^{* *}$ & $-0.338^{* * *}$ & $-0.249^{* *}$ & $-0.238^{* *}$ \\
\hline Grip strength & -0.051 & $-0.160^{*}$ & -0.142 & $0.233^{* *}$ & $-0.203^{*}$ & -0.063 & $-0.212^{* *}$ \\
\hline Knee extension strength & 0.154 & $-0.288^{* * *}$ & $-0.301^{* * *}$ & $0.242^{* *}$ & $-0.347^{* * *}$ & $-0.170^{*}$ & $-0.325^{* * *}$ \\
\hline Knee extension endurance & 0.108 & $-0.253^{* *}$ & $-0.296^{* * *}$ & $0.223^{* *}$ & $-0.308^{* * *}$ & -0.058 & $-0.223^{* *}$ \\
\hline
\end{tabular}

${ }^{*} P<0.05,{ }^{* *} P<0.01$, and ${ }^{* * *} P<0.001$. CA: categories achieved; IPS: index of postural stability; MoCA: Montreal Cognitive Assessment; OLST: one-leg standing time; PEN: perseverative errors of Nelson; QIDS: Quick Inventory of Depressive Symptomatology Self-Report; SAS: Starkstein Apathy Scale; TMT-A: Trail Making Test part A; TMT-B: Trail Making Test part B; TUG: Timed Up and Go Test; WCST: Wisconsin Card Sorting Test.

TABLE 4: Correlation between cognitive function test and olfactory identification, balance capability, and muscle strength in the type 2 diabetes group.

\begin{tabular}{|c|c|c|c|c|c|c|c|}
\hline & $\mathrm{MoCA}$ & TMT-A & TMT-B & WCST (CA) & WCST (PEN) & QIDS & SAS \\
\hline Open Essence & $0.264^{*}$ & -0.140 & $-0.244^{*}$ & 0.014 & -0.028 & -0.129 & -0.078 \\
\hline OLST (eyes open) & 0.149 & $-0.378^{* *}$ & $-0.239^{*}$ & 0.202 & -0.227 & 0.121 & -0.091 \\
\hline OLST (eyes closed) & $0.264^{*}$ & $-0.372^{* *}$ & $-0.315^{* *}$ & $0.291^{*}$ & -0.180 & 0.032 & -0.123 \\
\hline TUG & -0.124 & 0.175 & $0.264^{*}$ & -0.124 & $0.300^{*}$ & -0.090 & 0.152 \\
\hline IPS & $0.415^{* * *}$ & -0.187 & $-0.436^{* * *}$ & $0.374^{* *}$ & $-0.352^{* *}$ & -0.036 & 0.044 \\
\hline Grip strength & -0.104 & -0.076 & -0.011 & $0.334^{* *}$ & $-0.308^{* *}$ & 0.060 & -0.147 \\
\hline Knee extension strength & -0.016 & -0.164 & -0.132 & 0.179 & $-0.279^{*}$ & 0.029 & -0.149 \\
\hline Knee extension endurance & 0.055 & $-0.239^{*}$ & $-0.260^{*}$ & 0.189 & $-0.272^{*}$ & 0.078 & -0.123 \\
\hline
\end{tabular}

${ }^{*} P<0.05,{ }^{* *} P<0.01$, and ${ }^{* * *} P<0.001$. CA: categories achieved; IPS: index of postural stability; MoCA: Montreal Cognitive Assessment; OLST: one-leg standing time; PEN: perseverative errors of Nelson; QIDS: Quick Inventory of Depressive Symptomatology Self-Report; SAS: Starkstein Apathy Scale; TMT-A: Trail Making Test part A; TMT-B: Trail Making Test part B; TUG: Timed Up and Go Test; WCST: Wisconsin Card Sorting Test.

TABLE 5: Correlation between cognitive function test, olfactory identification test, balance capability tests, and muscle strength tests in the control group.

\begin{tabular}{lccccccc}
\hline & MoCA & TMT-A & TMT-B & WCST (CA) & WCST (PEN) & QIDS & SAS \\
\hline Open essence & -0.075 & -0.133 & 0.067 & 0.093 & -0.083 & -0.111 & 0.106 \\
OLST (eyes open) & 0.092 & -0.217 & -0.114 & 0.069 & -0.008 & -0.114 & $\mathbf{- 0 . 2 2 9}$ \\
OLST (eyes closed) & -0.026 & -0.050 & 0.151 & -0.013 & 0.007 & -0.197 & -0.057 \\
TUG & 0.056 & 0.109 & -0.111 & -0.006 & 0.003 & 0.095 & 0.062 \\
IPS & 0.159 & $\mathbf{- 0 . 2 5 0}$ & -0.146 & 0.086 & -0.121 & -0.142 & -0.205 \\
Grip strength & -0.146 & -0.176 & $\mathbf{- 0 . 2 1 9 ^ { * }}$ & 0.121 & -0.092 & -0.117 & $-\mathbf{0 . 2 3 8 ^ { * }}$ \\
Knee extension strength & -0.038 & -0.204 & $\mathbf{- 0 . 2 5 8 ^ { * }}$ & 0.180 & $\mathbf{- 0 . 2 5 6}^{*}$ & -0.090 & -0.216 \\
Knee extension endurance & -0.039 & -0.154 & $\mathbf{- 0 . 2 2 3}^{*}$ & 0.209 & $\mathbf{- 0 . 2 7 2}^{*}$ & -0.033 & $-\mathbf{0 . 2 4 0}^{*}$ \\
\hline
\end{tabular}

${ }^{*} P<0.05,{ }^{* *} P<0.01$, and ${ }^{* * *} P<0.001$. CA: categories achieved; IPS: index of postural stability; MoCA: Montreal Cognitive Assessment; OLST: one-leg standing time; PEN: perseverative errors of Nelson; QIDS: Quick Inventory of Depressive Symptomatology Self-Report; SAS: Starkstein Apathy Scale; TMT-A: Trail Making Test part A; TMT-B: Trail Making Test part B; TUG: Timed Up and Go Test; WCST: Wisconsin Card Sorting Test.

associated with MoCA, TMT-A and -B, and CA and PEN in WCST, and knee extension strength had an independent association with SAS.

\section{Discussion}

The aim of this study was at examining the associations between cognitive function and olfactory and physical func- tioning in middle-aged persons with and without T2D and at determining whether olfactory and physical functions could be biomarkers for early detection of cognitive impairment. It is also aimed at examining whether precise balance and muscle strength assessments using a stabilometer and a torque machine could be useful in predicting cognitive function in middle-aged adults. There were four major findings in the current study. First, results of all measurements of 
TABLE 6: Multiple linear regression analyses of cognitive functions in the entire participant group.

(a)

\begin{tabular}{|c|c|c|c|c|}
\hline & \multicolumn{2}{|c|}{$\mathrm{MoCA}^{*}$} & \multicolumn{2}{|c|}{$\mathrm{TMT} \mathrm{A}^{* *}$} \\
\hline & $\beta$ & $P$ & $\beta$ & $P$ \\
\hline Age & -0.061 & 0.466 & 0.034 & 0.700 \\
\hline Male & -0.102 & 0.301 & -0.043 & 0.675 \\
\hline Education $>12 \mathrm{y}$ & -0.043 & 0.590 & -0.035 & 0.679 \\
\hline Diabetes & -0.196 & 0.083 & -0.014 & 0.907 \\
\hline Hypertension & -0.019 & 0.809 & 0.149 & 0.070 \\
\hline ApoE $\varepsilon 4$ carrier & 0.040 & 0.584 & 0.033 & 0.668 \\
\hline Body mass index & 0.006 & 0.949 & -0.082 & 0.415 \\
\hline Open essence & 0.142 & 0.062 & -0.161 & 0.043 \\
\hline IPS & 0.329 & $<0.001$ & -0.260 & 0.006 \\
\hline Knee extension strength & -0.036 & 0.758 & -0.202 & 0.103 \\
\hline
\end{tabular}

(b)

\begin{tabular}{|c|c|c|c|c|}
\hline & \multicolumn{2}{|c|}{ TMT-B $^{* *}$} & \multicolumn{2}{|c|}{ WCST (CA) } \\
\hline & $\beta$ & $P$ & $\beta$ & $P$ \\
\hline Age & 0.092 & 0.268 & -0.125 & 0.170 \\
\hline Male & 0.089 & 0.367 & 0.144 & 0.180 \\
\hline Education $>12 \mathrm{y}$ & 0.093 & 0.244 & 0.044 & 0.617 \\
\hline Diabetes & 0.099 & 0.376 & -0.107 & 0.379 \\
\hline Hypertension & 0.070 & 0.372 & 0.100 & 0.241 \\
\hline ApoE $\varepsilon 4$ carrier & 0.022 & 0.762 & 0.042 & 0.596 \\
\hline Body mass index & -0.114 & 0.238 & 0.092 & 0.377 \\
\hline Open essence & -0.092 & 0.225 & 0.056 & 0.495 \\
\hline IPS & -0.324 & $<0.001$ & 0.319 & 0.001 \\
\hline Knee extension strength & -0.239 & 0.044 & 0.012 & 0.926 \\
\hline
\end{tabular}

(c)

\begin{tabular}{|c|c|c|c|c|}
\hline & \multicolumn{2}{|c|}{ WCST $(\mathrm{PEN})^{* *}$} & \multicolumn{2}{|c|}{ QIDS $^{* *}$} \\
\hline & $\beta$ & $P$ & $\beta$ & $P$ \\
\hline Age & 0.105 & 0.229 & -0.190 & 0.031 \\
\hline Male & -0.141 & 0.169 & -0.158 & 0.126 \\
\hline Education $>12 \mathrm{y}$ & -0.044 & 0.599 & -0.033 & 0.696 \\
\hline Diabetes & 0.215 & 0.066 & 0.290 & 0.014 \\
\hline Hypertension & -0.058 & 0.480 & 0.058 & 0.479 \\
\hline ApoE $\varepsilon 4$ carrier & -0.001 & 0.987 & 0.194 & 0.012 \\
\hline Body mass index & -0.094 & 0.348 & 0.038 & 0.703 \\
\hline Open essence & -0.080 & 0.307 & -0.155 & 0.052 \\
\hline IPS & -0.253 & 0.007 & -0.141 & 0.134 \\
\hline Knee extension strength & -0.097 & 0.429 & 0.030 & 0.811 \\
\hline
\end{tabular}

(d)

\begin{tabular}{lrr}
\hline & \multicolumn{1}{c}{ SAS } & $P$ \\
\hline Age & $\beta$ & $\mathbf{0 . 0 0 1}$ \\
Male & $\mathbf{- 0 . 3 0 2}$ & 0.886 \\
Education $>12 y$ & -0.015 & 0.224 \\
\hline
\end{tabular}


TABLE 6: Continued.

\begin{tabular}{lrr}
\hline & $\beta$ & SAS \\
\hline Diabetes & 0.173 & 0.145 \\
Hypertension & 0.019 & 0.817 \\
ApoE \&4 carrier & -0.005 & 0.952 \\
Body mass index & -0.108 & 0.288 \\
Open essence & -0.020 & 0.806 \\
IPS & -0.093 & 0.323 \\
Knee extension strength & $\mathbf{- 0 . 3 0 9}$ & $\mathbf{0 . 0 1 4}$ \\
\hline
\end{tabular}

${ }^{*}$ Cubed variables and ${ }^{* *} \log$-transformed variables are used for the analyses. ApoE: apolipoprotein E; CA: categories achieved; IPS: index of postural stability; MoCA: Montreal Cognitive Assessment; PEN: perseverative errors of Nelson; QIDS: Quick Inventory of Depressive Symptomatology Self-Report; SAS: Starkstein Apathy Scale; TMT-A: Trail Making Test part A; TMT-B: Trail Making Test part B; WCST: Wisconsin Card Sorting Test.

cognitive function, odor identification, balance capabilityrelated variables, and muscle strength-related variables in middle-aged persons with T2D were significantly worse than those in the age-matched controls. Second, balance capability measured by IPS was independently associated with all of the measured cognitive function tests except for QIDS and SAS in the entire cohort and the T2D group. Third, knee extension strength was significantly associated with SAS in the entire cohort and in the T2D group. Fourth, results of the odor identification test were independently associated with TMT-A in the entire cohort and with MoCA and TMT-B in the T2D group.

4.1. Cognitive Function and Physical Function in MiddleAged Persons with T2D. Previous reports showed decreased memory, attention, executive function, and information processing ability and increased prevalence of depression and apathy even in middle-aged patients with T2D [35-37]. In addition, decreased olfactory function, balance capability, and lower limb muscle strength have been shown in those with T2D [38-40]. Although sex and years of education differed significantly between the T2D group and the control group, those findings are consistent with our results; global cognition (MoCA), processing speed (TMT-A), executive function (TMT-B), cognitive flexibility (CA in WCST), perseveration (PEN in WCST), depressive symptoms (QIDS), motivation (SAS), odor identification, balance capability, and muscle strength in the T2D group were significantly worse than those in the control group. Those findings were also consistent even when the two participant groups were matched for age, sex, and years of education (Table 8). Although the proportion of ApoE $\varepsilon 4$ carriers was significantly higher in the control group than in the T2D group, both cognitive function and physical performance were significantly reduced in persons with T2D compared to nondiabetic individuals, suggesting that interventions to prevent cognitive and physical decline may be needed even in middle-aged persons with T2D.

4.2. Relationship between Cognitive Function and Physical Function. In the entire cohort of the current study, balance capability assessed by IPS was significantly associated with global cognition, processing speed, executive function, $\operatorname{cog}$ - nitive flexibility, and perseveration after adjustment for confounding factors. It was shown that the score of the MoCA was significantly associated with OLST with eyes open in community dwelling elderly persons [41]. Also, OLST with eyes open was reported to be significantly associated with memory, processing speed, and executive function in individuals aged 45 to 85 years (mean age 62.9 years) [42]. Tangen et al. reported that balance ability assessed with the Balance Evaluation Systems Test deteriorated in conjunction with increasing severity of cognitive impairment, especially in executive function, in individuals with subjective cognitive impairment, MCI, and $\mathrm{AD}$ [43]. Using a stabilometer, it was shown that the length of anteroposterior postural sway [44] or mean velocity of postural sway at the COP [45] was increased with the severity of cognitive impairment in elderly persons. Moreover, the sway path length was significantly correlated with supratentorial cerebrospinal fluid volume, white matter hyperintensities volume, and the Dementia Rating Scale [46].

There are few reports on the association between balance capability and cognitive function in T2D. Smith and colleagues showed that in elderly persons with T2D, executive function and reduced postural stability under dual task conditions were worse compared with those in age-matched controls [47]. In the current study, the IPS was the best balancing test in comparison with other balancing tests in terms of showing the broadest association with the cognitive domains measured (data not shown). The IPS is characterized by high correlation with the Berg balance scale, high reproducibility, and not having a ceiling effect [33]. In addition, the degree of difficulty in the IPS may have been suitable compared with other balance tests in middle-aged individuals.

There have been several reports on the association between cognitive function and lower limb muscle function [41, 48-50]. A study of community-dwelling older adults with no apparent cognitive impairment showed that handgrip strength, leg strength, sit-to-stand ratio, gait speed, and one-leg standing time were all significantly associated with MoCA scores in multiple regression analysis [41]. It was also reported that knee extension strength was significantly associated with executive function as assessed with the digit symbol substitution test after adjusting for 
TABLE 7: Multiple linear regression analyses of the cognitive function stratified according to the presence or absence of diabetes.

(a)

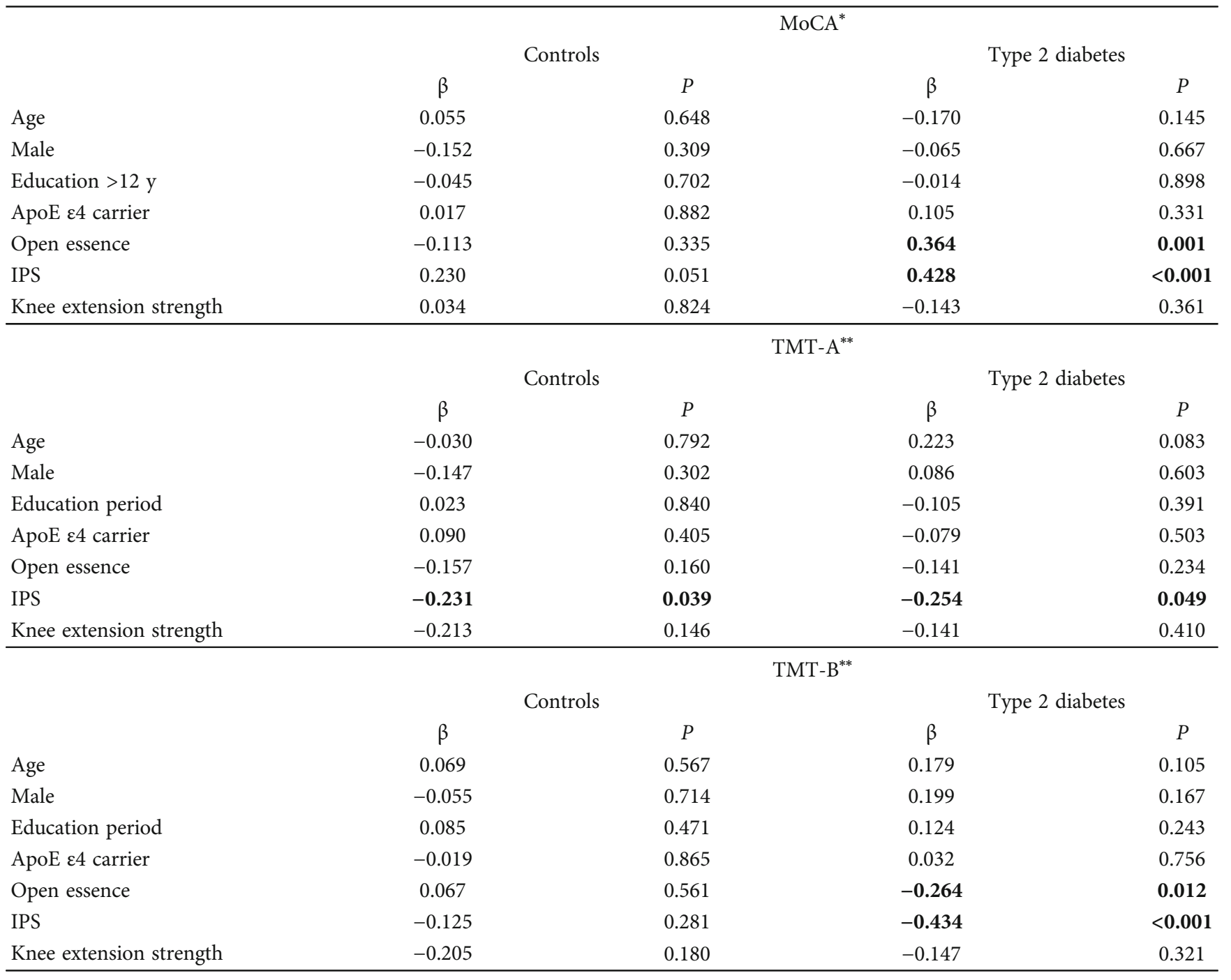

(b)

\begin{tabular}{|c|c|c|c|c|}
\hline & \multicolumn{4}{|c|}{ WCST (CA) } \\
\hline & \multicolumn{2}{|c|}{ Controls } & \multicolumn{2}{|c|}{ Type 2 diabetes } \\
\hline & $\beta$ & $P$ & $\beta$ & $P$ \\
\hline Age & -0.077 & 0.526 & -0.220 & 0.086 \\
\hline Male & 0.178 & 0.240 & 0.190 & 0.252 \\
\hline Education $>12 \mathrm{y}$ & 0.020 & 0.865 & 0.030 & 0.806 \\
\hline ApoE $\varepsilon 4$ carrier & 0.031 & 0.784 & 0.077 & 0.516 \\
\hline Open essence & 0.082 & 0.485 & 0.058 & 0.625 \\
\hline IPS & 0.116 & 0.323 & 0.379 & 0.004 \\
\hline \multirow[t]{4}{*}{ Knee extension strength } & 0.027 & 0.863 & -0.121 & 0.481 \\
\hline & \multicolumn{4}{|c|}{$\mathrm{WCST}(\mathrm{PEN})^{* *}$} \\
\hline & \multicolumn{2}{|c|}{ Controls } & \multicolumn{2}{|c|}{ Type 2 diabetes } \\
\hline & $\beta$ & $P$ & $\beta$ & $P$ \\
\hline Age & 0.211 & 0.072 & 0.060 & 0.645 \\
\hline Male & -0.189 & 0.193 & -0.174 & 0.309 \\
\hline
\end{tabular}


TABLE 7: Continued.

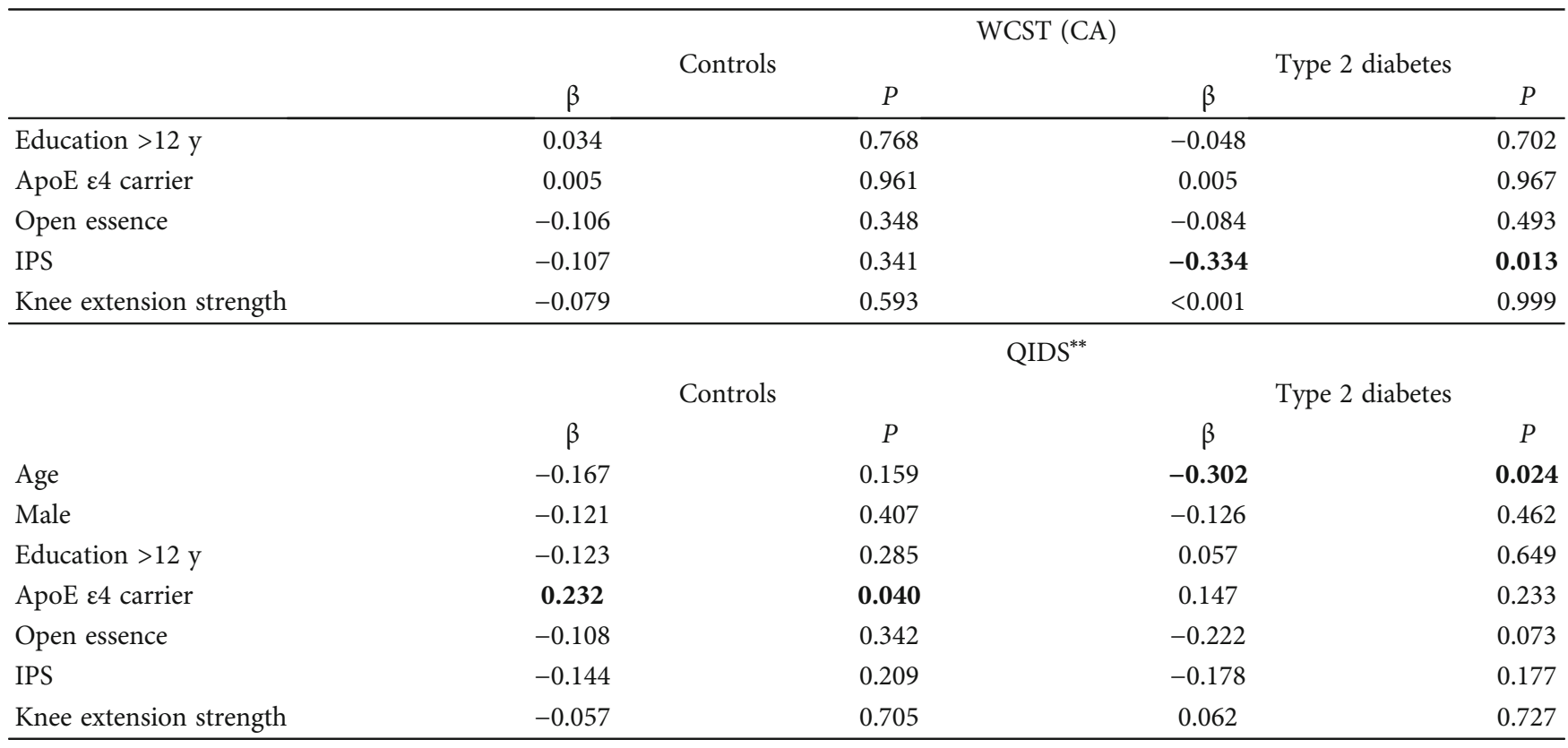

(c)

\begin{tabular}{|c|c|c|c|c|}
\hline & \multicolumn{4}{|c|}{ SAS } \\
\hline & \multicolumn{2}{|c|}{ Controls } & \multicolumn{2}{|c|}{ Type 2 diabetes } \\
\hline & $\beta$ & $P$ & $\beta$ & $P$ \\
\hline Age & -0.246 & 0.037 & -0.299 & 0.024 \\
\hline Male & -0.169 & 0.243 & 0.137 & 0.419 \\
\hline Education $>12 \mathrm{y}$ & -0.025 & 0.828 & -0.112 & 0.371 \\
\hline ApoE $\varepsilon 4$ carrier & -0.018 & 0.869 & -0.002 & 0.989 \\
\hline Open Essence & 0.081 & 0.471 & -0.075 & 0.538 \\
\hline IPS & -0.194 & 0.086 & $<0.001$ & 0.999 \\
\hline Knee extension strength & -0.166 & 0.264 & -0.375 & 0.035 \\
\hline
\end{tabular}

${ }^{*}$ Cubed variables and ${ }^{* *} \log$-transformed variables are used for the analyses. ApoE: apolipoprotein E; CA: categories achieved; IPS: index of postural stability; MoCA: Montreal Cognitive Assessment; PEN: perseverative errors of Nelson; QIDS: Quick Inventory of Depressive Symptomatology Self-Report; SAS: Starkstein Apathy Scale; TMT-A: Trail Making Test part A; TMT-B: Trail Making Test part B; WCST: Wisconsin Card Sorting Test.

confounding factors in individuals aged 60 years or older [48]. Steves and colleagues reported that leg power was significantly associated with both a 10-year cognitive decline and subsequent total grey matter volume in female twins [49]. A prospective study showed that physical activity in persons with apathy was significantly lower than that in persons without apathy and that apathy was associated with a decline in physical performance in elderly persons [50]. In the current study, knee extension strength was significantly associated with executive function and motivation after adjusting for confounding factors and was a better determinant of executive function and motivation than knee extension endurance (data not shown). No studies have examined the relationship between cognitive function and both knee extension strength and knee extension endurance. Further research on this topic is needed. The significant association between SAS and knee extension strength in the current study may indicate that low physical activity due to apathy causes low muscle strength.
4.3. Relationship between Cognitive Function and Olfactory Function. A study using positron emission tomography showed that the hippocampus, orbitofrontal cortex, amygdala, parietal cortex, insula, cerebellum, right temporal cortex, and parietal cortex were activated in odor discrimination and memory [51]. In persons with T2D, activation of the left hippocampus and left parahippocampus in response to odor stimuli was significantly reduced as was functional brain connectivity in the right inferior and middle orbitofrontal cortex compared with healthy individuals [52]. Although few reports have examined the relationship between olfactory function and cognitive function in middle-aged individuals, olfactory function has been suggested to be significantly associated with several cognitive domains $[52,53]$. Zhang et al. reported that olfactory behavior scores were significantly correlated with MoCA, word fluency, and executive function in persons with T2D but only significantly correlated with episodic memory in the control group [52]. Schubert et al. showed that olfactory 
TABLE 8: Comparison of cognitive function tests, odor identification tests, and physical function tests in age-, sex-, and years of educationmatched cohort.

\begin{tabular}{|c|c|c|c|}
\hline & $\begin{array}{l}\text { Controls } \\
(\mathrm{n}=44)\end{array}$ & $\begin{array}{l}\text { Type } 2 \text { diabetes } \\
(\mathrm{n}=44)\end{array}$ & $P$ \\
\hline Age (y) & $53 \pm 6$ & $53 \pm 7$ & 0.949 \\
\hline Female, n (\%) & $25(57)$ & $25(57)$ & 1.000 \\
\hline Education (y) & $14(12-16)$ & $14(12-16)$ & 0.313 \\
\hline ApoE $\varepsilon 4$ allele carrier, $\mathrm{n}(\%)$ & $13(30)$ & $4(9)$ & 0.014 \\
\hline \multicolumn{4}{|l|}{ Cognitive function } \\
\hline MoCA (points) & $27(26-28)$ & $25(22-26)$ & $<0.001$ \\
\hline TMT-A (s) & $25.0(21.9-29.8)$ & $32.1(26.5-38.4)$ & 0.001 \\
\hline TMT-B (s) & $60.0(49.2-77.8)$ & $85.6(60.6-108.5)$ & $<0.001$ \\
\hline \multicolumn{4}{|l|}{ WCST } \\
\hline CA (points) & $4(3-5)$ & $2(1-5)$ & 0.028 \\
\hline PEN (points) & $4(2-7)$ & $10(4-16)$ & $<0.001$ \\
\hline QIDS (points) & $3(2-6)$ & $5(4-8)$ & $<0.001$ \\
\hline SAS (points) & $9 \pm 5$ & $13 \pm 6$ & $<0.001$ \\
\hline \multicolumn{4}{|l|}{ Olfactory function } \\
\hline Open Essence (points) & $10(9-11)$ & $9(8-10)$ & 0.010 \\
\hline \multicolumn{4}{|l|}{ Physical function } \\
\hline OLST (eyes open) (sec) & $120(120-120)$ & $120(40-120)$ & $<0.001$ \\
\hline OLST (eyes closed) (s) & $20(9-29)$ & $7(4-16)$ & $<0.001$ \\
\hline TUG (s) & $5.0 \pm 0.7$ & $6.1 \pm 0.7$ & $<0.001$ \\
\hline IPS & $1.94(1.83-2.02)$ & $1.64(1.43-1.78)$ & $<0.001$ \\
\hline Grip strength (kg) & $31(26-41)$ & $26(21-40)$ & 0.046 \\
\hline Knee extension strength $(\mathrm{Nm} / \mathrm{kg})$ & $214 \pm 43$ & $164 \pm 48$ & $<0.001$ \\
\hline Knee extension endurance $(J)$ & $1081(899-1416)$ & $890(707-1179)$ & 0.006 \\
\hline
\end{tabular}

Data are mean \pm SD or median (interquartile range). CA: categories achieved; IPS: index of postural stability; MoCA: Montreal Cognitive Assessment; OLST: one-leg standing time; PEN: perseverative errors of Nelson; QIDS: Quick Inventory of Depressive Symptomatology Self-Report; SAS: Starkstein Apathy Scale; TMT-A: Trail Making Test part A; TMT-B: Trail Making Test part B; TUG: Timed Up and Go Test; WCST: Wisconsin Card Sorting Test.

impairment was significantly associated with poor performance on the TMT-A, TMT-B, and Grooved Pegboard in middle-aged adults [53]. The current study showed that odor identification was independently associated with TMT-A and had a tendency to be associated with MoCA and QIDS after adjustment for confounding factors in the entire cohort. Furthermore, the odor identification score was independently associated with MoCA and TMT-B in the T2D group but not in the control group. The results of the current study were consistent with the report of Zhang et al. [52]. Because the sample size of the control group in this study was smaller and performance on the TMT-A and TMT-B was better compared with a previous report [53], it is possible that there was no significant association between odor identification scores and results of the TMTA and TMT-B. Impairment of olfactory identification could be a surrogate marker of decreased frontal lobe function, which is responsible for information processing and executive functions [54].

4.4. Clinical Implications. The current study showed that several cognitive domains were significantly associated with odor identification, balance capability, and lower limb muscle strength. These physical tests are noninvasive and low cost. Testing of these physical abilities may identify groups at high risk for cognitive decline and allow for early detection and interventions. In particular, in multiple linear regression analyses, IPS was the strongest risk factor among a wide range of cognitive function domains compared to other risk factors. Therefore, IPS may be useful in predicting cognitive decline in those with and without T2D.

\section{Limitations}

This study has several limitations. First, the presence of organic brain diseases such as asymptomatic cerebral infarction could not be ruled out because the participants did not undergo magnetic resonance imaging. Second, the T2D group had a significantly shorter duration of education and included a significantly lower proportion of women and ApoE $\varepsilon 4$ carriers compared with the control group. Although we conducted multiple linear regression analyses, we cannot rule out the possibility that the effects of those imbalances were not fully corrected for. Third, this study did not consider the effects of antidiabetic drugs on cognitive function. Several antidiabetic drugs have been reported to affect cognitive function and olfactory function in animal studies and in human trials [55-59]. The inclusion of each 
antidiabetic drug as an explanatory variable instead of sex had no effect on the association between olfactory and physical function and cognitive function, except that metformin abolished the significance of knee extension strength in SAS (data not shown). In addition, the small sample size did not allow us to include those drugs in the model simultaneously without eliminating other possible risk factors for dementia. Finally, since this is a cross-sectional study with a small number of cases, it is not clear whether odor identification, balance capability, or lower muscle strength can predict future cognitive decline. It is necessary to conduct a longitudinal study with a larger study population.

\section{Conclusions}

In conclusion, middle-aged persons with T2D had lower cognitive function, olfactory function, balance capability, and lower extremity muscle strength than the nondiabetic controls. Odor identification, balance capability assessed with a stabilometer, and knee extension strength assessed by a torque machine were independent risk factors for $\operatorname{cog}$ nitive decline in the middle-aged study participants with T2D. These findings could be useful for early detection of cognitive decline.

\section{Data Availability}

The data used to support the findings of this study are available from the corresponding author upon request.

\section{Conflicts of Interest}

The authors declare no conflict of interest.

\section{Acknowledgments}

We would like to thank Takumi Tsubaki and Yuuki Tanabe of the Department of Rehabilitation Medicine, University of Tsukuba Hospital, for their cooperation in the measurement of physical function. This work was supported by JSPS KAKENHI Grant number 18K18443.

\section{References}

[1] Alzheimer's Disease International, World Alzheimer Report 2015: the global impact of dementia: an anAlysIs of prevalence, incidence, cost \& trends, 2015.

[2] M. Vaz and S. Silvestre, "Alzheimer's disease: Recent treatment strategies," European Journal of Pharmacology, vol. 887, p. $173554,2020$.

[3] R. C. Petersen, P. S. Aisen, L. A. Beckett et al., "Alzheimer's disease neuroimaging initiative (ADNI): clinical characterization," Neurology, vol. 74, no. 3, pp. 201-209, 2010.

[4] M. Canevelli, G. Grande, E. Lacorte et al., "Spontaneous reversion of mild cognitive impairment to normal cognition: a systematic review of literature and meta-analysis," Journal of the American Medical Directors Association, vol. 17, no. 10, pp. 943-948, 2016.
[5] G. Livingston, A. Sommerlad, V. Orgeta et al., "Dementia prevention, intervention, and care," The Lancet, vol. 390, no. 10113 , pp. $2673-2734,2017$.

[6] G. J. Biessels, S. Staekenborg, E. Brunner, C. Brayne, and P. Scheltens, "Risk of dementia in diabetes mellitus: a systematic review," The Lancet Neurology, vol. 5, no. 1, pp. 64-74, 2006.

[7] T. Ohara, Y. Doi, T. Ninomiya et al., "Glucose tolerance status and risk of dementia in the community: the Hisayama study," Neurology, vol. 77, no. 12, pp. 1126-1134, 2011.

[8] M. R. Rizzo, R. Marfella, M. Barbieri et al., "Relationships between daily acute glucose fluctuations and cognitive performance among aged type 2 diabetic patients," Diabetes Care, vol. 33, no. 10, pp. 2169-2174, 2010.

[9] C. Kim, J. H. Sohn, M. U. Jang et al., “Association between visit-to-visit glucose variability and cognitive function in aged type 2 diabetic patients: a cross-sectional study," PLoS One, vol. 10, no. 7, article e0132118, 2015.

[10] S. L. C. Geijselaers, S. J. S. Sep, C. D. A. Stehouwer, and G. J. Biessels, "Glucose regulation, cognition, and brain MRI in type 2 diabetes: a systematic review," The Lancet Diabetes \& Endocrinology, vol. 3, no. 1, pp. 75-89, 2015.

[11] M. V. F. Silva, C. . M. G. Loures, L. C. V. Alves, L. C. de Souza, K. B. G. Borges, and M. . G. Carvalho, "Alzheimer's disease: risk factors and potentially protective measures," J Biomed Sci, vol. 26, no. 1, p. 33, 2019.

[12] D. R. Roalf, M. J. Moberg, B. I. Turetsky et al., "A quantitative meta-analysis of olfactory dysfunction in mild cognitive impairment," Journal of Neurology, Neurosurgery, and Psychiatry, vol. 88, no. 3, pp. 226-232, 2017.

[13] A. M. Kotecha, A. Corrêa, K. Fisher, and J. Rushworth, “Olfactory dysfunction as a global biomarker for sniffing out Alzheimer's disease: a meta-analysis," Biosensors (Basel), vol. 8, no. 2, p. $41,2018$.

[14] H. J. Jung, I. S. Shin, and J. E. Lee, "Olfactory function in mild cognitive impairment and Alzheimer's disease: a meta-analysis," Laryngoscope, vol. 129, no. 2, pp. 362-369, 2019.

[15] R. S. Wilson, S. E. Arnold, J. A. Schneider, Y. Tang, and D. A. Bennett, "The relationship between cerebral Alzheimer's disease pathology and odour identification in old age," Journal of Neurology, Neurosurgery, and Psychiatry, vol. 78, no. 1, pp. 30-35, 2007.

[16] L. Bahureksa, B. Najafi, A. Saleh et al., "The impact of mild cognitive impairment on gait and balance: a systematic review and meta-analysis of studies using instrumented assessment," Gerontology, vol. 63, no. 1, pp. 67-83, 2017.

[17] A. R. Zammit, A. Robitaille, A. M. Piccinin, G. Muniz-Terrera, and S. M. Hofer, "Associations between aging-related changes in grip strength and cognitive function in older adults: a systematic review," The Journals of Gerontology Series A, Biological Sciences and Medical Sciences, vol. 74, no. 4, pp. 519-527, 2019.

[18] N. T. Aggarwal, R. S. Wilson, T. L. Beck, J. L. Bienias, and D. A. Bennett, "Motor dysfunction in mild cognitive impairment and the risk of incident Alzheimer disease," Archives of Neurology, vol. 63, no. 12, pp. 1763-1769, 2006.

[19] Q. Tian, S. M. Resnick, and S. A. Studenski, "Olfaction is related to motor function in older adults," The Journals of Gerontology Series A, Biological Sciences and Medical Sciences, vol. 72, no. 8, pp. 1067-1071, 2017.

[20] S. J. Kim, M. J. Windon, and S. Y. Lin, "The association between diabetes and olfactory impairment in adults: a 
systematic review and meta-analysis," Laryngoscope Investig Otolaryngol, vol. 4, no. 5, pp. 465-475, 2019.

[21] H. Sanke, T. Mita, H. Yoshii et al., "Relationship between olfactory dysfunction and cognitive impairment in elderly patients with type 2 diabetes mellitus," Diabetes Research and Clinical Practice, vol. 106, no. 3, pp. 465-473, 2014.

[22] E. Guerrero-Berroa, R. Ravona-Springer, A. Heymann et al., "Decreased motor function is associated with poorer cognitive function in elderly with type 2 diabetes," Dementia and Geriatric Cognitive Disorders Extra, vol. 4, no. 1, pp. 103112, 2014.

[23] C. Barbiellini Amidei, A. Fayosse, J. Dumurgier et al., "Association between age at diabetes onset and subsequent risk of dementia," JAMA, vol. 325, no. 16, pp. 1640-1649, 2021.

[24] C. L. Craig, A. L. Marshall, M. Sjöström et al., "International physical activity questionnaire: 12-country reliability and validity," Medicine and Science in Sports and Exercise, vol. 35, no. 8, pp. 1381-1395, 2003.

[25] Z. S. Nasreddine, N. A. Phillips, V. Ã.@. Bédirian et al., “The Montreal Cognitive Assessment, MoCA: a brief screening tool for mild cognitive impairment," Journal of the American Geriatrics Society, vol. 53, no. 4, pp. 695-699, 2005.

[26] G. Gagnon, K. T. Hansen, S. Woolmore-Goodwin et al., "Correcting the MoCA for education: effect on sensitivity," The Canadian Journal of Neurological Sciences, vol. 40, no. 5, pp. 678-683, 2013.

[27] C. R. Bowie and P. D. Harvey, "Administration and interpretation of the Trail Making Test," Nature Protocols, vol. 1, no. 5, pp. 2277-2281, 2006.

[28] S. Kobayashi, "Wisconsin Card Sorting Test (WCST)," Nippon Rinsho, vol. 61, Supplement 9, pp. 344-349, 2003.

[29] A. J. Rush, M. H. Trivedi, H. M. Ibrahim et al., "The 16-item quick inventory of depressive symptomatology (QIDS), clinician rating (QIDS-C), and self-report (QIDS-SR): a psychometric evaluation in patients with chronic major depression," Biological Psychiatry, vol. 54, no. 5, pp. 573-583, 2003.

[30] S. E. Starkstein, H. S. Mayberg, T. J. Preziosi, P. Andrezejewski, R. Leiguarda, and R. G. Robinson, "Reliability, validity, and clinical correlates of apathy in Parkinson's disease," The Journal of Neuropsychiatry and Clinical Neurosciences, vol. 4, no. 2, pp. 134-139, 1992.

[31] F. Okutani, K. Hirose, T. Kobayashi, H. Kaba, and M. Hyodo, "Evaluation of "Open Essence" odor-identification test card by application to healthy volunteers," Auris, Nasus, Larynx, vol. 40, no. 1, pp. 76-80, 2013.

[32] D. Podsiadlo and S. Richardson, "The timed "Up \& Go": a test of basic functional mobility for frail elderly persons," Journal of the American Geriatrics Society, vol. 39, no. 2, pp. 142148, 1991.

[33] Y. Suzuki, S. Yatoh, H. Suzuki et al., "Age-dependent changes in dynamic standing-balance ability evaluated quantitatively using a stabilometer," Journal of Physical Therapy Science, vol. 30, no. 1, pp. 86-91, 2018.

[34] J. E. Hixson and D. T. Vernier, "Restriction isotyping of human apolipoprotein $\mathrm{E}$ by gene amplification and cleavage with HhaI.," Journal of Lipid Research, vol. 31, no. 3, pp. 545-548, 1990.

[35] P. Palta, A. L. C. Schneider, G. J. Biessels, P. Touradji, and F. Hill-Briggs, "Magnitude of cognitive dysfunction in adults with type 2 diabetes: a meta-analysis of six cognitive domains and the most frequently reported neuropsychological tests within domains," Journal of the International Neuropsychological Society: JINS, vol. 20, no. 3, pp. 278-291, 2014.

[36] D. G. Bruce, G. P. Casey, V. Grange et al., "Cognitive impairment, physical disability and depressive symptoms in older diabetic patients: the Fremantle Cognition in Diabetes Study," Diabetes Research and Clinical Practice, vol. 61, no. 1, pp. 5967, 2003.

[37] D. G. Bruce, M. E. Nelson, J. L. Mace, W. A. Davis, T. M. E. Davis, and S. E. Starkstein, "Apathy in older patients with type 2 diabetes," The American Journal of Geriatric Psychiatry: Official Journal of the American Association for Geriatric Psychiatry, vol. 23, no. 6, pp. 615-621, 2015.

[38] E. Gouveri, M. Katotomichelakis, H. Gouveris, V. Danielides, E. Maltezos, and N. Papanas, "Olfactory dysfunction in type 2 diabetes mellitus: an additional manifestation of microvascular disease?," Angiology, vol. 65, no. 10, pp. 869-876, 2014.

[39] T. Nomura, T. Ishiguro, M. Ohira, H. Oka, and Y. Ikeda, "Isometric knee extension force in Japanese type 2 diabetic patients without apparent diabetic polyneuropathy: data from the multicenter survey of the isometric lower extremity strength in type 2 diabetes study," SAGE open Medicine, vol. 7, 2019.

[40] M. M. Vaz, G. C. Costa, J. G. Reis, W. M. Junior, F. J. Albuquerque de Paula, and D. C. Abreu, "Postural control and functional strength in patients with type 2 diabetes mellitus with and without peripheral neuropathy," Archives of Physical Medicine and Rehabilitation, vol. 94, no. 12, pp. 2465-2470, 2013.

[41] K. Narazaki, E. Matsuo, T. Honda, Y. Nofuji, K. Yonemoto, and S. Kumagai, "Physical fitness measures as potential markers of low cognitive function in Japanese communitydwelling older adults without apparent cognitive problems," Journal of Sports Science and Medicine, vol. 13, no. 3, pp. 590-596, 2014.

[42] N. Demnitz, D. B. Hogan, H. Dawes et al., "Cognition and mobility show a global association in middle- and late- adulthood: Analyses from the Canadian Longitudinal Study on Aging," Gait \& Posture, vol. 64, pp. 238-243, 2018.

[43] G. G. Tangen, K. Engedal, A. Bergland, T. A. Moger, and A. M. Mengshoel, "Relationships between balance and cognition in patients with subjective cognitive impairment, mild cognitive impairment, and Alzheimer disease," Physical Therapy, vol. 94, no. 8, pp. 1123-1134, 2014.

[44] M. Leandri, S. Cammisuli, S. Cammarata et al., "Balance features in Alzheimer's disease and amnestic mild cognitive impairment," Journal of Alzheimer's Disease : JAD, vol. 16, no. 1, pp. 113-120, 2009.

[45] T. Deschamps, O. Beauchet, C. Annweiler, C. Cornu, and J. B. Mignardot, "Postural control and cognitive decline in older adults: position versus velocity implicit motor strategy," Gait \& Posture, vol. 39, no. 1, pp. 628-630, 2014.

[46] E. V. Sullivan, J. Rose, T. Rohlfing, and A. Pfefferbaum, "Postural sway reduction in aging men and women: relation to brain structure, cognitive status, and stabilizing factors," Neurobiology of Aging, vol. 30, no. 5, pp. 793-807, 2009.

[47] M. A. Smith, J. E. Else, L. Paul et al., "Functional living in older adults with type 2 diabetes: executive functioning, dual task performance, and the impact on postural stability and motor control," Journal of Aging and Health, vol. 26, no. 5, pp. 841859, 2014.

[48] W. L. Chen, T. C. Peng, Y. S. Sun et al., "Examining the association between quadriceps strength and cognitive performance 
in the elderly," Medicine (Baltimore), vol. 94, no. 32, article e1335, 2015.

[49] C. J. Steves, M. M. Mehta, S. H. D. Jackson, and T. D. Spector, "Kicking back cognitive ageing: leg power predicts cognitive ageing after ten years in older female twins," Gerontology, vol. 62 , no. 2, pp. 138-149, 2016.

[50] M. J. Henstra, D. Rhebergen, M. L. Stek et al., "The association between apathy, decline in physical performance, and falls in older persons," Aging Clinical and Experimental Research, vol. 31, no. 10, pp. 1491-1499, 2019.

[51] I. Savic, B. Gulyas, M. Larsson, and P. Roland, "Olfactory functions are mediated by parallel and hierarchical processing," Neuron, vol. 26, no. 3, pp. 735-745, 2000.

[52] Z. Zhang, B. Zhang, X. Wang et al., "Altered odor-induced brain activity as an early manifestation of cognitive decline in patients with type 2 diabetes," Diabetes, vol. 67, no. 5, pp. 994-1006, 2018.

[53] C. R. Schubert, K. J. Cruickshanks, M. E. Fischer et al., "Odor identification and cognitive function in the Beaver Dam Offspring Study," Journal of Clinical and Experimental Neuropsychology, vol. 35, no. 7, pp. 669-676, 2013.

[54] P. Yuan and N. Raz, "Prefrontal cortex and executive functions in healthy adults: a meta-analysis of structural neuroimaging studies," Neuroscience and Biobehavioral Reviews, vol. 42, pp. 180-192, 2014.

[55] C. Palleria, C. Leporini, F. Maida et al., "Potential effects of current drug therapies on cognitive impairment in patients with type 2 diabetes," Frontiers in Neuroendocrinology, vol. 42, pp. 76-92, 2016.

[56] H. Umegaki, "Therapeutic potential of antidiabetic medications in the treatment of cognitive dysfunction and dementia," Drugs \& Aging, vol. 33, no. 6, pp. 399-409, 2016.

[57] T. Cukierman-Yaffe, H. C. Gerstein, H. M. Colhoun et al., "Effect of dulaglutide on cognitive impairment in type 2 diabetes: an exploratory analysis of the REWIND trial," The Lancet Neurology, vol. 19, no. 7, pp. 582-590, 2020.

[58] G. Lietzau, W. Davidsson, C. G. Östenson et al., "Type 2 diabetes impairs odour detection, olfactory memory and olfactory neuroplasticity; effects partly reversed by the DPP-4 inhibitor Linagliptin," Acta Neuropathol Commun, vol. 6, no. 1, p. 14, 2018.

[59] Z. Zhang, B. Zhang, X. Wang et al., "Olfactory dysfunction mediates adiposity in cognitive impairment of type 2 diabetes: insights from clinical and functional neuroimaging studies," Diabetes Care, vol. 42, no. 7, pp. 1274-1283, 2019. 